\title{
Tumor angiogenesis in predicting the survival of patients with stage $I$ lung cancer
}

\author{
Baris Medetoglu, MD, ${ }^{\mathrm{a}}$ Mehmet Zeki Gunluoglu, MD, ${ }^{\mathrm{a}}$ Adalet Demir, MD, ${ }^{\mathrm{a}}$ Huseyin Melek, MD, \\ Nur Buyukpinarbasili, MD, ${ }^{\mathrm{b}}$ Neslihan Fener, MD, ${ }^{\mathrm{b}}$ and Seyyit Ibrahim Dincer, $\mathrm{MD}^{\mathrm{a}}$
}

Objective: The effects of angiogenesis on survival were assessed by measuring the tumor microvessel density
and vascular endothelial growth factor expression in patients with resected stage I non-small cell lung carcinoma.

\begin{abstract}
Methods: The study population included 141 patients who underwent complete resection for stage pT1 and T2 N0 M0 tumors between 1999 and 2007. Lobectomy and pneumonectomy were performed in 131 and 10 patients, respectively. Tumor specimens were analyzed immunohistochemically for staining with anti-CD105 antibody to determine tumor microvessel density and anti-vascular endothelial growth factor antibody to determine the vascular endothelial growth factor expression level. Univariate and multivariate analyses were performed for factors influencing patients' survival.
\end{abstract}

Results: The overall 5 -year survival was calculated as $68 \%$, with rates of $76.9 \%$ for patients with T1 disease and $66.2 \%$ for patients with $\mathrm{T} 2$ disease $(P=.4)$. The vascular endothelial growth factor expression rate was $94.3 \%$ for patients with stage I non-small cell lung carcinoma. Vascular endothelial growth factor expression did not influence survival $(P=.9)$. The median microvessel density of the tumors measured based on the level of CD105 expression was 19.8. The effect of microvessel density on survival was significant $(P=.02)$. The 5 -year survivals of patients with tumors with 20 or more microvessels and less than 20 microvessels were $76.8 \%$ and $56.1 \%$, respectively; this difference was highly significant $(P=.004)$. The microvessel density was determined as an independent factor influencing survival on multivariate analysis $(P=.03)$.

Conclusions: The level of vascular endothelial growth factor expression in tumors was not a successful predictor of survival in patients with resected stage I non-small cell lung carcinoma. A high microvessel density based on CD105 is a strong predictor of prognosis in these patients. (J Thorac Cardiovasc Surg 2010;140:996-1000)

Most patients with non-small cell lung cancer (NSCLC) who survive for a long time have tumors at TNM stage I or II at the time of initial diagnosis. However, despite potentially curative treatment, the 5-year survival is not yet satisfactory, even in these patients. ${ }^{1,2}$

Many factors considered to affect prognosis in patients with NSCLC have been and are still being investigated. Improvements in our understanding of the genetic and molecular basis of lung cancer in recent years have led to the discovery and assessment of new factors that might be of prognostic value. The level of neoangiogenesis in the tumor tissue is one of the factors assessed previously. ${ }^{3}$ The density of vascular structures in the tumor tissue can be determined by means of immunohistochemical staining of the tissue for the endothelial cell marker CD105 (endoglin). ${ }^{4}$ One of the factors known to affect the level of neoangiogenesis is vascular endothelial growth factor (VEGF), ${ }^{5}$ the level of

From the Departments of Thoracic Surgery ${ }^{\mathrm{a}}$ and Pathology, ${ }^{\mathrm{b}}$ Yedikule Teaching Hospital for Chest Diseases and Thoracic Surgery, Istanbul, Turkey.

Disclosures: None.

Received for publication Sept 10, 2009; revisions received June 25, 2010; accepted for publication July 6, 2010; available ahead of print Aug 16, 2010.

Address for reprints: Adalet Demir, MD, Yuzyil mah. Kisla Cad. Yesil zengibar sitesi,

A-3 Blok, D-9 Bagcilar, Istanbul, Turkey (E-mail: dradalet@hotmail.com).

$0022-5223 / \$ 36.00$

Copyright (c) 2010 by The American Association for Thoracic Surgery

doi:10.1016/j.jtcvs.2010.07.002 expression of which in tumor tissue can also be determined by using immunohistochemical staining. Several studies have examined the effects of tumor VEGF and CD105 expression in patients with NSCLC but yielded inconsistent results. Moreover, TNM stages of the patient populations were heterogeneous or the tumors were limited to only 1 histopathological type in most of these studies.

The present study was performed to assess the effects of the expression levels of the major neoangiogenesis markers CD105 and VEGF in tumor tissue, as measured with immunohistochemical methods, on the survival of patients with completely resected stage I NSCLC.

\section{MATERIALS AND METHODS}

Our clinical database was searched to find patients with stage I (T1 or T2 and N0 M0) NSCLC surgically treated between January 1999 and January 2007. During this period, 557 consecutive lung resections because of NSCLC were performed. One hundred sixty-nine patients with pathologically diagnosed stage I NSCLC were found (30\% of all of the patients). Patients with low-grade tumor $(n=8)$, those in whom sublobar $(n=5)$ or incomplete $(n=1)$ resection had been performed, and those with no appropriate tissue sample from their tumors $(\mathrm{n}=12)$ were excluded. Two patients who had died within the first 30 days postoperatively or in the hospital were also excluded because the operative mortality is related to the surgical procedure or functional limitations of the patients and was not relevant to this study. In total, 141 patients with $\mathrm{T} 1(\mathrm{n}=26)$ or $\mathrm{T} 2(\mathrm{n}=115)$ and N0 MO NSCLC were included in the study. Because this was a retrospective 


\section{Abbreviations and Acronyms \\ IQR = interquartile range \\ NSCLC $=$ non-small cell lung cancer \\ VEGF $=$ vascular endothelial growth factor}

study, institutional review board approval was not required. The scientific study committee of our hospital reviewed and approved the database.

The patients consisted of $15(10.6 \%)$ women and $126(89.4 \%)$ men at a median age of 59 years (interquartile range [IQR], 54-64 years). Mediastinoscopy was performed in $132(93.6 \%)$ patients as part of routine prethoracotomy mediastinal evaluation, and no mediastinal metastasis was detected. In other patients computed tomographic analysis of the thorax or positron emission tomographic analysis was used to eliminate the risk of mediastinal lymphatic metastasis. Neoadjuvant chemotherapy and radiotherapy were not provided. Systematic mediastinal lymphatic dissection was performed during thoracotomy along with appropriate lung resection. Lung resection consisted of lobectomy in 131 patients and pneumonectomy in 10 (who have T2 tumors) patients (Table 1). No patient received adjuvant therapy.

Resected specimens were sent to our pathology department for histopathological and immunohistochemical examination. Specimens were fixed in $10 \%$ formalin and embedded in paraffin. The longest diameter of each tumor was measured, and one or two $4-\mu$ m-thick sequential sections were obtained for each centimeter of the tumor from the areas that contained the most dense viable tumor tissue. Several sections were subjected to routine hematoxylin and eosin staining, and others were kept for immunohistochemical staining.

Tumors were staged after thoracotomy (pTNM) according to the 6th International System for Staging Lung Cancer developed by the American Joint Committee for Cancer in 1997. ${ }^{1}$ Histopathological tumor types were determined according to the classification of the World Health Organization. ${ }^{6}$ Histopathological tumor types and differentiations, T stages, and tumor sizes are shown in Table 1.

VEGF expression level and microvessel density (MVD) based on CD105 expression levels were determined by using immunohistochemical staining by 2 pathologists who were blind to the survival results.

\section{Immunohistochemical Staining}

For each patient, one section that contained the most dense tumor tissue was selected. Sections were taken onto adhesive (poly-L-lysine)-coated slides, deparaffinized through a graduated xylene and alcohol series, and then rehydrated in distilled water. Antigen retrieval was performed by adding citrate buffer and heating in a microwave oven. Sections were incubated in a $3 \%$ hydrogen peroxide solution to remove endogen peroxidase activity and washed with a phosphate-buffered saline solution. The UV blocking procedure was carried out to remove nonspecific immunoreactivity. Immunohistochemical staining for VEGF to highlight VEGF expression and for CD105 to highlight endothelial cells was performed by using a sensitive streptavidin-biotinylated peroxidase complex system. All of the procedures were performed in accordance with the antibody manufacturer's protocols. Sections were incubated with anti-VEGF mAb (Epitope Specific Rabbit Antibody, Vascular Endothelial Growth Factor, catalog no. RB-9031-R7) and anti-CD105 mAb (Rabbit Polyclonal Antibody, CD105/Endoglin, TGF- $\beta 1 /$ 3 Receptor, catalog no. RB-9291-R7) as primary antibodies (NeoMarkers; Lab Vision Corp, Fremont, Calif). Then antigen-antibody complexes were visualized with a biotin-labeled secondary antibody and streptavidin. After this procedure, amino ethyl-carbazole chromogen (ScyTek Laboratories, Inc, Logan, Utah) was applied to the sections. Then contrast staining was performed with Mayer's hematoxylin, and sections were covered with a water-based mounting medium (Aqueous-Mount, ScyTec).

\section{Evaluation of Tumor MVD}

The 3 most vascular areas (hot spots) within a section were selected for quantification of angiogenesis, and vessels labeled with the anti-CD105 $\mathrm{mAb}$ were counted under light microscopy at $400 \times$ magnification. Each single or connected endothelial cell stained red in these areas was counted as a microvessel. The average counts were recorded as the CD105-MVD for each case.

\section{Evaluation of VEGF Expression Level}

All tumor areas of the sections were examined, and the rate of tumor cells stained with anti-VEGF was determined. Staining intensity was also determined as a rate. Tumors were graded according to the rate of stained cells as follows: 0 for no stained cells, 1 for $1 \%$ to $25 \%, 2$ for $26 \%$ to $50 \%, 3$ for $51 \%$ to $75 \%$, and 4 for greater than $75 \%$ of the tumor cells stained. Staining intensity was also divided into 4 grades. Stained cells and staining intensity grades were combined to form a total VEGF score.

\section{Follow-up}

Patients were followed up with regard to survival or recurrence at 6-month intervals routinely. Additionally, patients were called by telephone to obtain further information during the preparation of this article. During follow-up, tumor recurrence was discovered in 35 patients. For T1 stage disease, no local recurrence was seen, but distant metastases were observed in 4 patients. Local recurrence and distant metastases occurred in 8 and 23 patients with T2 stage disease, respectively. The survival period was calculated by using the day of hospital discharge as the first day and the day of death or last contact as the final day. The median follow-up period was 47 months (IQR, 33-66 months). During the follow-up period, 44 patients died ( 6 with T1 and 38 with T2 tumors). Causes of death were known in 31 $(70.5 \%)$ patients; one of the patients with $\mathrm{T} 1$ disease had another primary cancer (breast cancer) and died, another patients with $\mathrm{T} 1$ disease died from distant metastasis, and 1 patient died from a cause unrelated to lung cancer. However, the cause of death could not be established in the other 3 patients with T1 NSCLC. Three patients with T1 disease who had tumor recurrences were still alive. Among 38 patients with T2 disease who died, cause of death was tumor recurrence in 25 . The cause of death was unrelated to the tumor in 3 patients and was not known in the remaining 10 patients. Six patients with T2 disease who had tumor recurrence were still alive.

\section{Statistical Analysis}

Cases were evaluated for demographic, surgical, and pathological variables, and the distributions of these variables were compared by using the $\chi^{2}$ or Fisher's exact tests. Correlations were determined by using the Spearman rank correlation test. Patients' survival was analyzed by using the Kaplan-Meier method.

Differences in survival were determined by using the log-rank test on univariate analysis, and prognostic factors with $P$ values of less than .1 were included in a multivariate analysis by using the Cox proportional hazards regression model.

\section{RESULTS}

Adenocarcinoma histopathologic type was more common $(50 \%, \mathrm{n}=13)$ in the $\mathrm{T} 1$ group compared with $\mathrm{T} 2$ group $(32 \%)$, and more $\mathrm{T} 1$ tumors $(26 \%)$ were observed among the patients with adenocarcinoma (vs $12 \%$ in the patients with squamous cell cancer, $P=.09$ ). The overall 5 -year survival of the patients was calculated as $68 \%$. The effects of histopathological type on survival were assessed, and the 5-year survival was found to be $75.1 \%$ for patients with squamous cell carcinoma and $56.8 \%$ for patients with 
TABLE 1. Prognostic factors revealed by means of univariate and multivariate analyses in patients with completely resected T1 N0 M0 and T2 N0 M0 non-small cell carcinoma of the lung $(n=141)$

\begin{tabular}{lccc}
\hline & Univariate & Multivariate & \\
& $\boldsymbol{P}$ value & $\boldsymbol{P}$ value & HR $(\mathbf{9 5} \% \mathbf{C I})$ \\
\hline Age & $\mathbf{. 0 2}$ & $\mathbf{. 0 0 3}$ & $1.06(1.02-1.11)$ \\
Sex & .21 & & \\
Tumor size & $\mathbf{. 0 9}$ & .13 & \\
pT classification & .4 & & \\
Histology (squamous & $\mathbf{. 0 4}$ & .22 & \\
$\quad$ vs adenocarcinoma) & & & \\
Tumor differentiation & .87 & & \\
Surgical procedure & .7 & & \\
Perineural invasion & .36 & & \\
Lymphatic vessel invasion & .42 & & \\
Blood vessel invasion & $\mathbf{. 0 0 6}$ & $\mathbf{. 0 1}$ & $0.45(0.24-0.84)$ \\
CD105 & $\mathbf{. 0 2}$ & $\mathbf{. 0 3}$ & $1.03(1-1.06)$ \\
VEGF & .91 & & \\
\hline
\end{tabular}

$H R$, Hazard ratio; $C I$, confidence interval; $V E G F$, vascular endothelial growth factor.

adenocarcinoma. Differences among groups were statistically significant $(P=.04)$. The 5-year survival was $76.9 \%$ for patients with stage $\mathrm{T} 1$ disease and $66.2 \%$ for those with stage T2 disease. However, this difference was not statistically significant $(P=.4)$. Sex, tumor differentiation, and type of lung resection did not affect survival. In addition, no significant differences were observed in the survivals of patients with and without lymph vessel or perineural invasion. However, univariate analysis revealed that age, tumor size, and invasion of the tumor to the tissue vasculature influenced survival (Table 1).

No VEGF-positive cells were observed in $8(5.7 \%)$ of the 141 tumors examined by using immunohistochemical staining. Thus the VEGF expression rate of stage I NSCLC was $94.3 \%$. The median rate of cells that stained positively with anti-VEGF antibody was 50\% (IQR, 40\%-70\%). AntiVEGF-stained cell rate, VEGF staining intensity, and total VEGF score did not influence survival at univariate analysis $(P=.9, P=.4$, and $P=.9$, respectively).

The mean MVD of the tumors determined by the level of CD105 expression was $19.8 \pm 9.8$, and survival was influenced by MVD $(P=.02)$. The 5-year survivals of patients with tumors having less than 20 microvessels or 20 or more microvessels were $76.8 \%$ and $56.1 \%$, respectively $(P=.004$, Figure 1). Tumor diameter did not influence survival in patients who had tumors with low vascularity $(<20$ microvessels, $P=.7$ ).

A significant correlation was observed between the VEGF expression level and the MVD of the tumors determined by means of CD105 $(P=.0001)$; however, the correlation was weak $(R=0.4)$.

Factors that were found to affect survival on univariate analysis $(P<.1$; ie, age, tumor size, histopathological tumor type, vascular invasion of the tumor, and CD105-assessed MVD) were used in a Cox regression analysis for multivar-

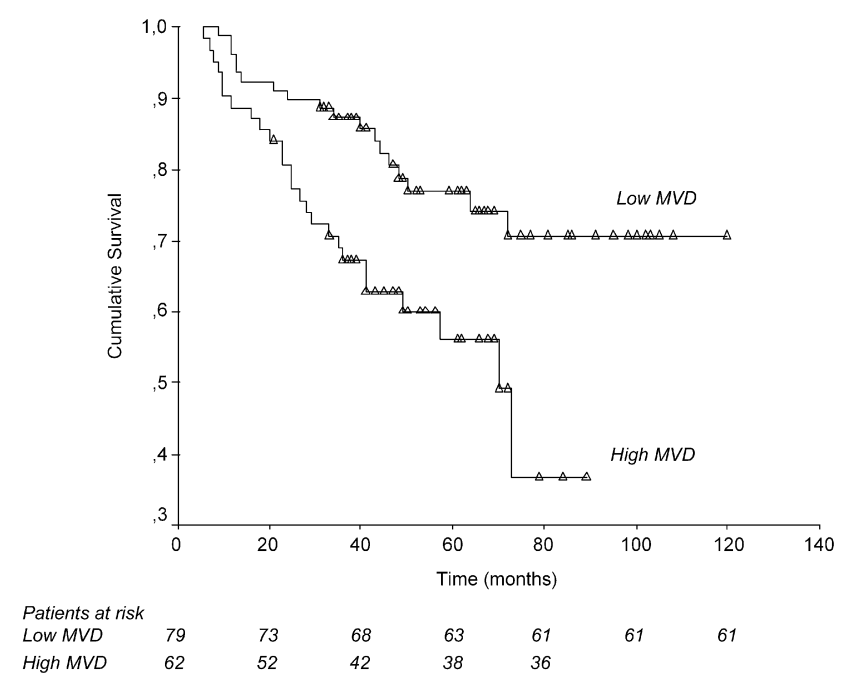

FIGURE 1. Survival curves of patients having non-small cell lung carcinoma with high or low microvessel density $(M V D)$.

iate analysis of the factors that might have affected survival. The analysis showed that MVD determined by the CD105 level was a factor that apparently and independently influenced survival, along with age and blood vessel invasion (Table 1).

Twenty-two (35.5\%) of the 62 patients with high CD105assessed MVD and $13(16.5 \%)$ of the 79 patients with low MVD experienced recurrence or metastasis. This difference in frequency was statistically significant $(P=.01)$.

\section{DISCUSSION}

Many patients with NSCLC experience and die of relapse, although surgical treatment is potentially curative. ${ }^{7}$ Staging helps to predict the overall survival of a group of patients, but predicting the prognosis of a specific patient with NSCLC is not that reliable. Thus new prognostic factors are required to determine the subgroup of patients in the same stage who have relatively poor prognosis.

Inclusion of patients with all stages of cancer in a study implemented to detect prognostic factors might impede the discovery of effective factors. The effects of these new factors can remain relatively weak compared with $\mathrm{T}, \mathrm{N}$, and $\mathrm{M}$ variables, which are known to strongly affect survival. We included patients with early T-stage tumors without lymph node or distant organ metastasis to minimize the effects of these primary survival markers. This was expected to uncover the effects of other potentially useful factors, and there is no any study specifically interested in the effect of angiogenesis in this subgroup of patients.

The angiogenesis level of the tumor showed a prominent effect on survival in some studies, ${ }^{3}$ and a high vascularity of the tumor was shown to indicate the survival tendency of tumor cells. ${ }^{8}$ Variables related to tumor angiogenesis have been investigated in many other types of cancer and have 
already been shown to be effective. ${ }^{9,10}$ The level of tumor angiogenesis was recently demonstrated to have an effect on the prognosis of patients with resected lung cancer. ${ }^{8,11,12}$ One method of determining the angiogenesis level of a tumor is to measure the microvessel density of the tumor (ie, to directly determine the number of vessels in the tumor); a greater number of microvessels signifies strong angiogenesis. ${ }^{9,13}$ Tumor MVD, determined either by panendothelial markers or CD105, has been shown to be an indicator of poor survival in various types of cancer $^{4,11}$ and also a poor prognostic factor in lung cancer. ${ }^{11}$ Koukourakis and colleagues ${ }^{13}$ concluded that tumor MVD is a factor independently affecting the prognosis of patients with lung cancer. CD105-assessed MVD, however, shows a significantly better correlation with survival than other factors. ${ }^{14}$ In the present study CD105-assessed MVD was also determined as a strong factor affecting the survival of patients with stage I NSCLC. The multivariate analysis also indicated that CD105-assessed MVD is an independent factor affecting survival. In addition, a high MVD was shown to be valuable for the detection of local recurrence and distant metastasis.

Measurement of the proangiogenic factor levels in tumor tissue or in the body fluids is a way to indirectly determine the level of tumor angiogenesis. VEGF is the most potent proangiogenic factor. ${ }^{5}$ Therefore the VEGF level in serum or tumor tissue has been used to detect angiogenesis and thus act as a prognostic indicator in patients with cancer. ${ }^{5}$ The VEGF level was determined based on the ratio of stained cells in several studies, and staining intensity of the cells was added as an additional factor in some other studies. Analysis is rather subjective, and staining is highly influenced by laboratory conditions. In the present study we determined both the ratio and the intensity; however, VEGF level did not influence survival. Most previous studies reported that the VEGF level is a poor prognostic factor of survival in patients with cancer. ${ }^{12,14}$ Some of the studies that examined VEGF levels in the tumorous masses of patients with NSCLC determined the VEGF level to be an independent factor that influenced patient survival. ${ }^{15,16}$ However, most studies indicated that a high VEGF level was associated with a high MVD or correlated with MVD. ${ }^{9,12,13,17}$ In this context VEGF is believed to decrease survival by promoting angiogenesis. In contrast, many studies failed to demonstrate an influence of VEGF level on the survival of patients with lung cancer. ${ }^{18,19}$ These studies indicated that the VEGF level lacked a reliable correlation with MVD. We determined a weak correlation between VEGF level and MVD. Although VEGF is the most important proangiogenic factor, it is only one of the factors regulating angiogenesis. The angiogenesis level of a tumor is under the control of various proangiogenic and antiangiogenic factors; the net result for angiogenesis is determined by the balance between these factors and thus the formation of the angiogenic switch.
An angiogenic switch will not form if strong antiangiogenic factors exist in the environment, despite increased expression of VEGF by the tumor. ${ }^{13}$ Thus high levels of VEGF in a tumor might not always lead to increased angiogenesis. These findings suggest that tumor MVD might be superior to VEGF alone for predicting patient survival, and the VEGF level might not be the most important factor determining MVD.

The survival of a patient with NSCLC depends on numerous factors, and accurately determining the prognosis of a specific patient is not possible. However, the angiogenesis level of the tumor is a useful factor that can be used in predicting survival of patients with stage I NSCLC. VEGF, a major factor that influences angiogenesis, is not a reliable predictor of the survival of these patients or their possibility of recurrence. However, CD105-assessed MVD of tumors in stage I NSCLC is a reliable indicator of a patient's prognosis and the possibility of local or distant recurrence of these tumors.

The present study also has some limitations. T stage was not found to be a significant prognostic factor. Also, the prognostic effect of the histopathological type disappeared on multivariate analysis. These results could be related to heterogeneous distribution. The rate of adenocarcinoma in the $\mathrm{T} 1$ tumor group and the rate of $\mathrm{T} 1$ tumor in the adenocarcinoma group were relatively high. Therefore these effects might have neutralized each other. Additionally, the effect of $T$ stage might not have been apparent because of small sample size (only 26 patients with $\mathrm{T} 1$ disease). A second limitation of the study is that we could not find the causes of death in some cases.

\section{References}

1. Mountain CF. Revisions in the international system for staging lung cancer Chest. 1997;111:1710-7.

2. Pairolero PC, Williams DE, Bergstralh EJ, Piehler JM, Bernatz PE, Payne WS. Postsurgical stage I bronchogenic carcinoma: morbid implications of recurrent disease. Ann Thorac Surg. 1984;38:331-8.

3. Buccheri G, Ferrigno D. Prognostic factors in lung cancer: tables and comments. Eur Respir J. 1994; 7:1350-64.

4. Kumar S, Ghellal A, Li C, Byrne G, Haboubi N, Wang JM, et al. Breast carcinoma: vascular density determined using CD105 antibody correlates with tumor prognosis. Cancer Res. 1999;59:856-61.

5. Tanaka F, Ishikawa S, Yanagihara K, Miyahara R, Kawano Y, Li M, et al. Expression of angiopoietins and its clinical significance in non-small cell lung cancer. Cancer Res. 2002;62:7124-9.

6. Travis WD, Colby TV, Corrin B, Shimosato Y, Brambilla E. Histological typing of tumours of lung and pleura. In: Sobin LH, ed. World Health Organization international classification of tumours. 3rd ed. Berlin: Springer-Verlag; 1999.

7. Tanoue LT, Matthay RA. Lung cancer: epidemiology and carcinogenesis. In: Shields TW, ed. General thoracic surgery. 5th ed. Philadelphia: Lippincott Williams \& Wilkins; 2000:1215-28.

8. Baillie R, Carlile J, Pendleton N, Schor AM. Prognostic value of vascularity and vascular endothelial growth factor expression in non-small cell lung cancer. J Clin Pathol. 2001;54:116-20.

9. Fontanini G, Vignati S, Boldrini L, Chinè S, Silvestri V, Lucchi M, et al. Vascular endothelial growth factor is associated with neovascularization and influences progression of non-small cell lung carcinoma. Clin Cancer Res. 1997;3:861-5.

10. Yang LY, Lu WQ, Huang GW, Wang W. Correlation between CD105 expression and postoperative recurrence and metastasis of hepatocellular carcinoma. $B M C$ Cancer. 2006;6:110. 
11. Macchiarini P, Fontanini G, Dulmet E, de Montpreville V, Chapelier AR, Cerrina J, et al. Angiogenesis: an indicator of metastasis in non-small cell lung cancer invading the thoracic inlet. Ann Thorac Surg. 1994;57:1534-9.

12. Bremnes RM, Camps C, Sirera R. Angiogenesis in non-small cell lung cancer: the prognostic impact of neoangiogenesis and the cytokines VEGF and bFGF in tumours and blood. Lung Cancer. 2006;51:143-58.

13. Koukourakis MI, Giatromanolaki A, Thorpe PE, Brekken RA, Sivridis E, Kakolyris S, et al. Vascular endothelial growth factor/KDR activated microvessel density versus CD31 standard microvessel density in non-small cell lung cancer. Cancer Res. 2000;60:3088-95.

14. Saad RS, Liu YL, Nathan G, Celebrezze J, Medich D, Silverman JF. Endoglin (CD105) and vascular endothelial growth factor as prognostic markers in colorectal cancer. Mod Pathol. 2004;17:197-203.

15. Yuan A, Yu CJ, Shun CT, Luh KT, Kuo SH, Lee YC, et al. Total cyclooxygenase-2 mRNA levels correlate with vascular endothelial growth factor mRNA levels, tumor angiogenesis and prognosis in non-small cell lung cancer patients. Int J Cancer. 2005;115:545-55.

16. O’Byrne KJ, Koukourakis MI, Giatromanolaki A, Cox G, Turley H, Steward WP, et al. Vascular endothelial growth factor, platelet-derived endothelial cell growth factor and angiogenesis in non-small-cell lung cancer. Br J Cancer. 2000;82:1427-32.

17. Mineo TC, Ambrogi V, Baldi A, Rabitti C, Bollero P, Vincenzi B, et al. Prognostic impact of VEGF, CD31, CD34, and CD105 expression and tumour vessel invasion after radical surgery for IB-IIA non-small cell lung cancer. J Clin Pathol. 2004;57:591-7.

18. Decaussin M, Sartelet H, Robert C, Moro D, Claraz C, Brambilla C, et al. Expression of vascular endothelial growth factor (VEGF) and its two receptors (VEGFR1-Flt1 and VEGF-R2-Flk1/KDR) in non-small cell lung carcinomas (NSCLCs): correlation with angiogenesis and survival. J Pathol. 1999;188:369-77.

19. Takahama M, Tsutsumi M, Tsujiuchi T, Kido A, Okajima E, Nezu K, et al. Frequent expression of the vascular endothelial growth factor in human nonsmall-cell lung cancers. Jpn J Clin Oncol. 1998;28:176-81. 